\title{
SUPERCONDUCTING FOCUSING QUADRUPOLES FOR HEAVY ION FUSION EXPERIMENTS*
}

\author{
G. Sabbi \#, A. Faltens, M. Leitner, A. Lietzke, P. Seidl, LBNL, Berkeley, CA \\ J. Barnard, S. Lund, N. Martovetsky, LLNL, Livermore, CA \\ C. Gung, J. Minervini, A. Radovinsky, J. Schultz, MIT-PSFC, Cambridge, MA \\ R. Meinke, Advanced Magnet Lab, Palm Bay, FL
}

\begin{abstract}
The Heavy Ion Fusion (HIF) Program is developing superconducting focusing magnets for both near-term experiments and future driver accelerators. In particular, single bore quadrupoles have been fabricated and tested for use in the High Current Experiment (HCX) at Lawrence Berkeley National Laboratory (LBNL). The next steps involve the development of magnets for the planned Integrated Beam Experiment (IBX) and the fabrication of the first prototype multi-beam focusing arrays for fusion driver accelerators. The status of the magnet $R \& D$ program is reported, including experimental requirements, design issues and test results.
\end{abstract}

\section{INTRODUCTION}

The HIF program is progressing through a series of physics and technology demonstrations leading to an IFE power plant [1]. A set of scaled experiments carried out during the past several years has confirmed theoretical calculations of space charge dominated beam transport. In order to determine if these results can be extended to the power levels required by HIF targets, experiments with high current beams are currently underway in the areas of injection, transport and final focus. The next phase will involve source-to-target experiments to demonstrate that all beam manipulations required by the driver can be carried out in an integrated manner, thus setting the basis for a demonstration power plant.

Efficiency requirements determine the use of superconducting quadrupoles for beam transport in most of the driver accelerator [2]. Superconducting magnets are also preferred for near term experiments, to gain operational experience and to better simulate the beam environment in a fusion driver. Magnet development for HIF is carried out by a collaboration of Lawrence Berkeley National Laboratory (LBNL), Lawrence Livermore National Laboratory (LLNL), MIT Plasma Science and Fusion Center, and Advanced Magnet Lab (AML). In this paper, the experimental requirements are discussed, and the magnet $R \& D$ status and plans are presented.

\footnotetext{
* Supported by the Office of Energy Research, US DOE, at LBNL and LLNL under contract numbers DE-AC03-76SF00098, W-7405-Eng-48, and at MIT under contract number DE-FC02-93-ER54186.

\# Tel: 510-495-2250, e-mail: GLSabbi@lbl.gov
}

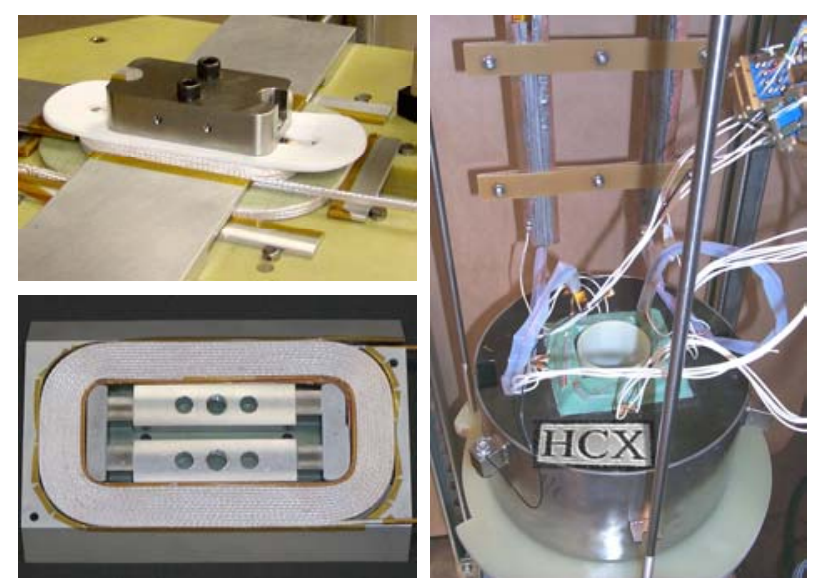

Fig. 1: Left: HCX optimized quadrupole coil fabrication. Right: Completed magnet undergoing test preparations.

\section{HIGH CURRENT EXPERIMENT}

HCX is designed to explore the physics of intense beams with driver-scale line-charge density $(0.2 \mu \mathrm{C} / \mathrm{m})$ and pulse duration $(\tau \geq 4 \mu \mathrm{s})$ [3]. Magnetic transport experiments in HCX will primarily investigate the effects due to electrons trapped in the potential well of the ion beam. A quadrupole gradient of $84.2 \mathrm{~T} / \mathrm{m}$ over a magnetic length of $10.1 \mathrm{~cm}$ and a coil aperture of $70 \mathrm{~mm}$ are required [4]. Following analysis and comparison of several design concepts, prototype quadrupoles of two different types were fabricated and tested [5]. A 2-layer racetrack design, developed by LLNL [6], was finally selected and further improved [7]. A prototype of the optimized design has been fabricated by AML and is ready for testing at LBNL (Fig 1). The coil ends were modified from continuous arcs to tight bends followed by straight segments. This layout improves the magnetic length relative to the physical coil length, and gives better field quality. However, some difficulties were encountered in winding the cable around the $6 \mathrm{~mm}$ radius. To address this problem, cable optimization studies were performed and a slight increase of the minimum bending 
radius is planned for future models. A change of the coil holder material from stainless steel to aluminum was also incorporated. The projected cost of aluminum holders in production is significantly lower than for stainless steel. However, experience with magnet fabrication showed that some design improvements are required to take into account larger deflections of the aluminum holder after applying pre-stress to the coils. Ultimately, the choice between the two materials will depend on the magnet test results. The structural tube used in the bore of previous prototypes to provide internal support to the coils was eliminated, to increase the space available to the beam for a given coil aperture. The superconducting strand was changed from SSC-outer to SSC-inner type, with lower copper fraction. The strand was redrawn from $0.808 \mathrm{~mm}$ to $0.648 \mathrm{~mm}$ for compatibility with the previous cable dimensions. The calculated short sample gradient is 132 $\mathrm{T} / \mathrm{m}$, with an effective magnetic length of $105.4 \mathrm{~mm}$ for a coil physical length of $125 \mathrm{~mm}$.
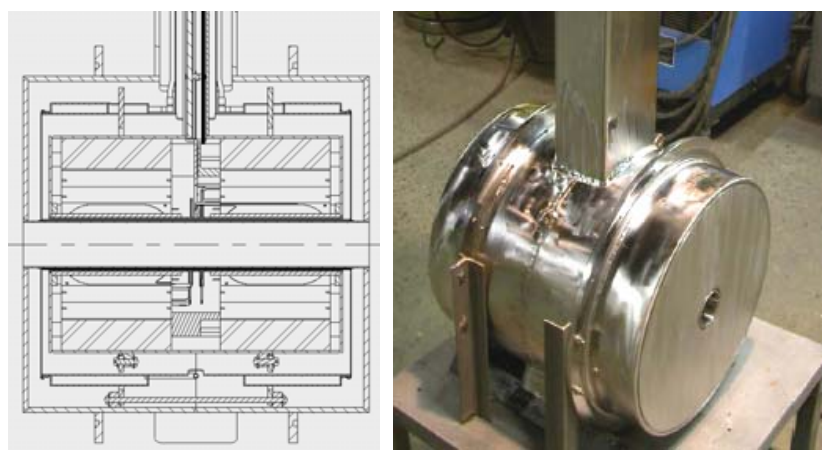

Fig 2: HCX focusing doublet design and fabrication.

A compact focusing doublet prototype, designed by LLNL, was fabricated by MIT and CVIP Inc. using two of the first-generation quadrupoles (Fig. 2). The cryostat design is compatible with the HCX lattice period of 45 $\mathrm{cm}$, and provides a warm axial gap between cryostat tanks, as required for induction acceleration, diagnostics and pumping ports. The coil leads and cryogen supplies are provided through a central chimney, to maximize the space for induction acceleration cores surrounding the beam transport line. In order to minimize the radial space between the beam pipe and the LHe vessel in the magnet bore, special low-emissivity aluminized stainless steel foils are used for radiation shields [8]. However, high vacuum and low surface contamination are required for the foil to work properly. This excludes the combined use of MLI superinsulation in the same vacuum space, due to outgassing. Application of the foil in all areas of the cryostat, and in particular close to welds, proved labor intensive. The use of separate vacuum chambers has been proposed to address this problem. The first cryostated unit will be cold tested at MIT to check quench performance and measure the heat loads in different configurations before installation in HCX. Based on this experience, a second focusing doublet will be designed and fabricated using optimized HCX magnets.

\section{INTEGRATED BEAM EXPERIMENT}

The purpose of the IBX is to integrate in a single experiment all beam manipulations required in a driver, including injection, acceleration, compression, bending and final focus, at significant line-charge density [9]. The beam will be accelerated to an energy of about $10 \mathrm{MeV}$ in a magnetic transport section consisting of 50 lattice periods (100 quadrupoles). The machine design is in progress, and the magnet parameters have not yet been finalized. In particular, the option of using resistive (pulsed) quadrupoles or steady state superconducting quadrupoles is still being evaluated. The magnet parameter range being considered is: clear bore $40-80$ $\mathrm{mm}$, operating gradient 40-120 T/m, magnetic length 8-14 $\mathrm{cm}$. For a given integrated strength, high gradients over a short magnet length are preferred, to increase the space for accelerating gaps, cryostat terminations and beam diagnostics. The design must accommodate dipole steering coils and may include a cold beam pipe (at $4.5 \mathrm{~K}$ ) with a $77 \mathrm{~K}$ baffle-like beam screen [10].

The HCX racetrack quadrupole is well matched to IBX requirements with simple modifications. For a clear bore of $80 \mathrm{~mm}$ and a gradient of $60-70 \mathrm{~T} / \mathrm{m}$, a design based on a single coil layer has been proposed, allowing significant cost savings (Fig. 3). The coil parameters are very similar to the HCX outer coils. The short sample gradient is in the range of 70-80 T/m, depending on the conductor properties (critical current density, packing and copper fraction). With respect to the dimensions quoted in Fig. 3, the gradient could be increased by reducing the radial space allocations. The thickness of the cold bore tube could be decreased, pending demonstration of the coil performance in the absence of internal mechanical support (HCX prototype test). Some aperture gains can also be obtained with more efficient designs of the beam screen and the dipole steering coils.

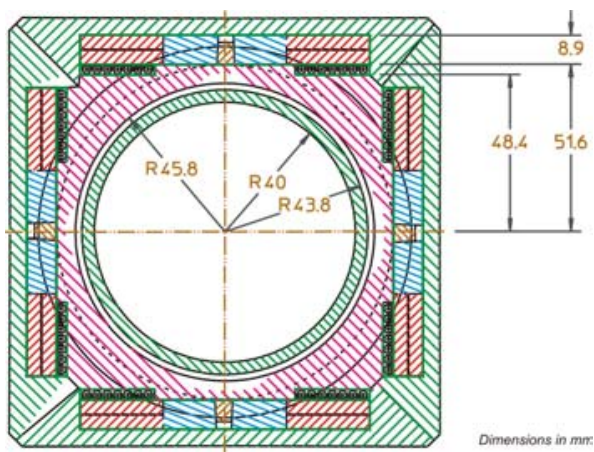

Fig. 3: Racetrack design for IBX (dimensions in mm).

An alternative two-layer coil of the HCX type would satisfy IBX magnet designs aiming at small aperture and very high gradient. Assuming some optimization of the radial space allocation in the magnet bore, the coil aperture corresponding to a $40 \mathrm{~mm}$ clear bore is $60 \mathrm{~mm}$, a small extrapolation with respect to the HCX. For a $60 \mathrm{~mm}$ coil aperture, a short sample gradient of $160-170 \mathrm{~T} / \mathrm{m}$ can be achieved. 
While the HCX racetrack design appears well suited to IBX, other approaches may be considered. Possible alternatives include a simple shell $(\cos 2 \theta)$ design of the RHIC type [11]. A significant difference in magnetic efficiency between shell and racetrack coils is not expected for very short magnets, but shell-type coils are radially more compact in a single-bore configuration. A compact magnet results in a more effective acceleration system. The RHIC arc quadrupole is close to the IBX aperture and gradient specifications, and is already optimized for low cost and robust performance. However, contrary to IBX, no strong constraint on the coil end design was present in RHIC (1.1 m magnetic length). In order to obtain very compact ends and meet gradient specifications, the magnet would have to be redesigned using a two-layer coil with a narrow cable. The development of a new shell design requires expensive tooling and experimental verification of the magnet performance by fabrication and test of several prototypes.

\section{MULTIPLE BEAM EXPERIMENTS}

Following IBX, experiments with multiple beams will be performed, to study magnetic coupling effects at high energy and provide the basis for a high average fusion power Engineering Test Facility (ETF). Superconducting quadrupole arrays must be developed for this application. The design challenges include minimization of the transverse size (to limit the size and cost of the induction accelerator cores), implementation of special edge coils to adjust the field in outer cells and terminate the magnetic flux, and maximization of the quadrupole gradient [12].

Since a very large number of cells is required in the fusion driver, economy of fabrication is also a primary consideration. Racetrack coils are simple to fabricate and well suited to the square cell layout of the array. They can be arranged back-to-back improving flux sharing, and they require less structural support since the outwards components of the magnetic force are balanced between cells. Neighboring coils can in fact be combined with reduction of the number of parts, conductor joints, and inductance. However, further R\&D is required to simplify the fabrication methods with respect to those presently used for HCX. Cost reductions may result from the use of kapton insulation without epoxy impregnation, and by applying the bladders and keys technology [13] to prestress to the entire array in a single step.

Other innovative approaches to magnet design are being considered. Coil designs using round cables individually supported by grooved cylinders or plates have been actively explored by AML [14]. Although the first prototypes did not perform as well as the racetrack coils, with further R\&D these issues may be resolved, and ultimately result in very cost-effective magnets. A new concept, again based on a round cable and grooved supports, but with a helical winding geometry, is also being developed [15].

Special edge coils are required to adjust the field in the boundary cells and terminate of the magnetic flux within the shortest radial distance from the array, avoiding magnetic coupling between the quadrupole array and the surrounding induction cores. While several 2D studies have been performed in the past, recent analysis has shown that finding acceptable solutions is significantly more difficult if end effects are considered. Preliminary results show that the use of an iron yoke may be beneficial in addressing these issues [16].

\section{SUMMARY}

Accelerators for fusion energy production will require arrays of superconducting quadrupoles to transport multiple beams in the accelerator and focus them on the target. The development of superconducting magnets for both near term experiments and the ultimate driver application is being actively pursued by the U.S. Heavy Ion Fusion Program. The main parameters and requirements have been discussed, and the $R \& D$ status and plans have been presented.

\section{REFERENCES}

[1] J. Barnard et al., "Progress in Heavy Ion Driven Inertial Fusion Energy: from Scaled Experiments to the Integrated Research Experiment," Proceedings of the 2001 Particle Accelerator Conference, Chicago, June 2001.

[2] W. Meier et al., "A 3.3 MJ, Rb+1 Driver Design based on an Integrated Systems Analysis," Nucl. Instr. and Meth. A 464 (2001) 433-439.

[3] P. Seidl et al., "The High Current Transport Experiment for Heavy Ion Inertial Fusion", this conference.

[4] S. Lund et al., "Characterization of Prototype Superconducting Quadrupoles for the High Current Experiment," HCX Magnet Note 01-0222-01, February 2001.

[5] A. Lietzke et al., "Development of Superconducting Quadrupoles for Heavy Ion Fusion", Proceedings of the 2001 Particle Accelerator Conference, Chicago, June 2001.

[6] N. Martovetsky, B. Manahan, "Focusing Magnets for HIF based on Racetracks," IEEE Trans. Appl. Superconductivity, 11 (1), pp 1506-1509, March 2001.

[7] A. Faltens et al., "Progress in the development of superconducting quadrupoles for heavy ion fusion", Laser and Particle Beams (2002), 20, 617-620.

[8] M. Larin et al., "Liquid Helium Cryopumps with low-emissivity Al film coatings and low helium consumption," J. Vac. Sci. Tech. A(13)5, 1995

[9] J. Barnard et al., "Integrated Experiments for Heavy Ion Fusion," Laser and Particle Beams, accepted for publication (2003).

[10] M. Leitner et al., "Technology Choices for the Integrated Beam Experiment (IBX)", $15^{\text {th }}$ Topical Meeting on the Technology of Fusion Energy, Washington, November 2002. To be published in Fusion Science and Technology.

[11] M. Anerella et al., "The RHIC Magnet System", NIM A 499 (2003) 280-315.

[12] G. Sabbi et al., "Parameters and Requirements of Superconducting Focusing Quadrupoles for Heavy Ion Fusion", Proccedings of the 2002 Applied Superconductivity Conference, Houston, TX, August 2002.

[13] S. Caspi et al., "The Use of Pressurized Bladders for Stress Control of Superconducting Magnets", IEEE Trans. Appl. Supercond. Vol. 11, No. 1, March 2001.

[14] R. Meinke et al., "Development of Quadrupole Arrays for Heavy Ion Fusion”, IEEE Trans. Appl. Supercond. 10 (1), March 2000.

[15] R. Meinke et al., "Modulated Double-Helix Quadrupole Magnets", Proccedings of the 2002 Applied Super-conductivity Conference, Houston, TX, August 2002.

[16] A. Radovinsky, "Four Quad Double Helical Array with an Iron Yoke. Proof of Principle", HCX-MIT-031703-01, March 2003. 